\title{
Die sogenannte chronische Metritis, ihre Ursachen und ihre Symptome.
}

\author{
Von
}

Dr. A. Theilhaber in München.

Abnorme Uterusblutungen, die durch ibre Menge, oder durch die lange Dauer, oder durch ihre häufige Wiederkehr die Patientinnen schwächen, sind bekanntlich ein ausserordentlich häufiges Symptom bei unterleibskranken Frauen. Die häufigsten Ursachen solcher Blutungen sind:

1. Veränderungen in der Schleimhaut des Uterus. Hierher gehören die Schleimhautpolypen, zurückgebliebene Eihautreste nach Entbindungen, Aborten u. s. w., ab und za vielleicht auch einmal diffuse Entzündungsprocesse der Schleimhaut.

2. Veränderungen im Mesometrium (in der sogenannten Muskelschicht des Uterus) und zwar:

a) ungenügende Ausbildung der Musculatur (in den ersten Jahren nach der Pubertät);

b) Atrophie der Musculatur in den vorgerückteren Lebensjahren bei und nach consumirenden Krankheiten etc.;

c) Wucherungen des Bindegewebes und regressive Metamorphose in der Musculatur nach zahlreichen Entbindungen, nach langjährigen Adnexerkrankungen etc.;

d) interstitielles Oedem in der Musculatur bei acuten und subacuten Erkrankungen der Adnexe.

3. Hyperämie des Uterus oder eines Theiles desselben.

a) bei reichlicher Entwicklung seiner Gefässe;

b) in Folge von Dilatation der Gefässe durch vasomotorische Einflüsse. a und b sind natürlich häufig combinirt.

4. Anwesenheit eines Fremdkörpers im Uterus (Placentarrest z. B.) oder eines als Fremdkörper wirkenden Tumors (z. B. eines 
submucösen Myomes), wodurch die vollständige Zusammenziehung der Musculatur des Vterus verhindert wird.

5 . Ulcerationsprocesse (carcinomatöse, tuberculöse Geschwüre u. s. w.).

Nach meinen Beobachtungen sind die häufigsten Ursachen der Uterusblutungen die in die Rubrik 2 und 3 fallenden: am häufigston haridelt es sich bei Menorrhagien und Metrorrhagien um Veränderungen im Mesometrium, combinirt mit Hyperämie des Uterus.

Schon vor $1 / 2$ Jahrhundert hatte Scanzoni die Auffassung vertreten, dass die weitaus häufigste Frauenkrankheit die "chronische Metritis" sei. Mittelst seiner Monographie über die chronische Metritis gelang es ihm auch, nahezu alle Frauenärzte zu veranlassen, seiner Lehre beizutreten. Dass die Auffassung ron Seanzoni sich nur wenige Jabrzehnte halten konnte, lag vor Allem daran, dass, wie Fritsch (D. Chir, von Billroth u. Lücke. 56. p. 300) mit Recht betont, der Abschnitt über die pathologische Anatomie in Scanzoni's Monographie von der chronisehen Metritis als der am wenigsten befriedigende erklärt werden muss. Scanzoni führt das an, was er bei Rokitansky, Förster, Nonat und Anderen fand. Scanzoni gesteht offen, dass ihm die Lust zu mikroskopischen Untersuchnngen vergangen sei, als zwei anerkannte A utoritäten den mikroskopischen Präparaten geradezu eine entgegengesetzte Deutung gegeben hatten. Seine Untersuchungen waren in Folge dessen nur makroskopischer Natur. Er constatirte, dass das hauptsächlichste anatomische Charakteristikum die Vergrösserung des Uterus ist und dieselbe, wenn auch durchaus nicht eine ganz gleichmässige, so doch eine allseitige ist. Ebenso wie Becquerel constatirte er sehr verschiedene Formen, fasste dieselben jedoch als zwei Stadien auf: Im ersten Stadium fände sich serös blutige oder serös faserstoffige Infiltration des Gewebes mit starker Hyperämie des Uterus. Im zweiten Stadium fände sich allgemeine oder partielle Blutarmuth des Uterus, Trockenheit, Derbheit und Härte seines Gewebes, Verengerung der Gefässe. Gleichzeitig fände sich in diesem Stadium meist eine Bindegewebsneubildung. Der erste Autor, der nach Scanzoni wieder eingehendere Studien über die chronische Metritis machte, war Fritseh (l. c.). Seine Monographie ist voll trefflicher Beobachtungen und origineller Gedanken. Doch hatte er sie zu einer Zeit geschrieben (1885), in der es ausserordentlich schwierig, ja nahezu unmöglich war, sich Uteri zu verschaffen, deren Besitzerinnen an „chronischer 
Metritis" gelitten hatten. Damals wurden ja solche Uteri noch nicht exstirpirt. Seinen Arbeiten konnte deshalb selbstverständlich ebenfalls nicht genügend anatomisches Material zu Grunde gelegt werden. Er gesteht auch ein, dass diese Lücke von späteren Forschern ausgefüllt werden müsse. Er erklärt ausdrücklich, dass die mangelnde anatomische Untersuchung die Ursache der verschiedenartigen Anschauungen über das Wesen der chronischen Metritis sei. Während manche Autoren die Vergrösserung z. B. als Folge des Prolapses erklärten, sahen andere in derselben die Ursache des Vorfalles. In einzelnen Lehrbüchern wiederum käme der Name nchronische Metritis" überhaupt nicht vor. Es sei nur die Rede von Hyperämie, Bindegewebswucherung oder Congestionszuständen. "Aber", meint er (l. c. S. 41) „diese verschiedenen Anschauungen würden ja sehr vortheilhaft gewesen sein, wenn dadurch irgend Jemand veranlasst worden wäre, in ausführlicher Art anatomisch die Frage klar zu legen. Dies ist nie geschehen; man weiss ja nicht einmal, ob die anatomischen Bilder der chronischen Metritis von solchen Frauen stammen, welche im Leben an den Erscheinungen der chronischen Metritis gelitten haben. Irgend ein zufällig bei der Obduction gefundener, abnorm gross erscheinender Uterus wirde meist untersucht und das, was man fand, galt als das typische Bild der chronischen Metritis. An dieser Krankheit starb natürlich die Frau nicht und ein intercurrenter Tod hat schon durch die Circulationsänderung jedenfalls auf die Blutfülle des Uterus so wesentlichen Einfluss, dass Rückschlüsse unstatthaft sind. Man hat beim klinischen Bild wesentlich die Vergrösserung des Uterus als charakteristisch angenommen und glaubte nun, dass eo ipso jeder vergrösserte Uterus chronisch metritisch verändert sei. Gewiss sind hier Zweifel gerechtfertigt."

In Folge des Umstandes, dass auch Fritsch nicht in der Lage war, seine klinischen Beobachtungen durch einwandfreie anatomische Untersuchungen zu belegen, gelang es ihm nicht, die Lehre von der grossen Bedeutung der chronischen Metritis aufrecht $z u$ erhalten. Ein weiterer Umstand wirkte noch sehr ungünstig auf die Auffassung von der Bedeutung der Muskelveränderungen im Uterus, die zu hohe Meinung, die man in den letzten Jahrzehnten von der Bedentung der Bakterien für die Entstehung der verschiedensten Krankheiten hatte. Die Beeinflussung der Uteruserkrankungen durch Bakterien liess sich am leichtesten erklären, wenn man die Schleimhaut als den ursprünglichen Herd der Erkrankung, 
die Veränderungen in der Musculatur dagegen als die Folgeerscheinung der Schleimhauterkrankung ansah.

So bürgerte sich immer mehr der Glaube ein, dass bei den "entzündlichen" Erkrankungen des Uterus die "Endometritis" in der Regel das primäre, die "Mesometritis" secundär sei.

Die Untersuchungen, die in den letzten Jahren von mir, meinen Assistenten Dr. A. Neier und Dr. Hollinger und von dem Volontair meiner Klinik, v. Lorentz, an nun mehr als 100 Uteris gemacht wurden, führen zu den entgegengesetzten Schlüssen. Nennenswerthe diffuse Verdickungen der Schleimhaut des Uterus sind auch bei Frauen mit Metrorrhagien recht selten; auch mikroskopisch konnten wir bis jetzt typische Veränderungen der Schleimhaut, die in Causalnexus zu den Blutungen zu bringen sind, bis jetat nicht feststellen.

Wer heute sich mit Untersuchungen über die chronische Metritis beschäftigt, ist in einer weit glücklicheren Lage, als s. Zt. Scanzoni und Fritseh. Auch noch beim Erscheinen von Fritsch's Monographie war die Totalexstirpation des Uterus ein Eingriff von nicht geringer Gefährlichkeit, so dass sie damals vorwiegend bei carcinomatösen Erkrankungen des Organs ausgeführt wurde. Heute hat die vaginale Totalexstirpation ihre Gefahr nahezu vollständig verloren; man ist gewiss berechtigt, bei schweren, auf andere Weise nicht zu beseitigenden Blutungen, auch bei solchen, die mit Tumorenbildung im Uterus nicht einhergehen, das kranke Organ zu entfernen. Auf Grund dieser Indication haben Prof. Klein und ich in den letzten Jahren je fünfmal die Totalexstirpation des Cterus ausgeführt. Herr Klein hatte dio grosse Liebenswürdigkeit, mir seine Cteri zur Verfügung zu stellen, wofür ich ihm nochmals meinen besten Dank ausspreche. Die Cteri wurden von einem Volontair meiner Klinik, Herrn von Lorentz, eingehend untersucht. Ueber das Ergebniss seincr Untersuchungen berichtet Herr von Lorentz ausführlich in diesom Arehiv. Mir seien zu diesem Thema nur einige Bemerkungen gestattet: In den Fällen, in denen ich den Uterus wegen Blutungen cxstirpirte, fand ich stets bei starken Blutungen (die nicht durch Tumorenbildung veranlasst waren) das Corpus uteri wesentlich verbreitert, vergrössert, manchmal so gross, dass man vor der Operation an die Möglichkeit einer Complication mit Myombildung denken musste; doch war die Grösse nicht immer zu allen Zeiten die gleiche. Namentlich während der prämenstruellen Zeit und 
während der Blutung fühlte sich der Uterus gewöhnlich grösser und schlaffer an. Es war dies zum Theil die Folge des vermehrten Blutgehaltes in dieser Zeit. Nach Beendigung der Blutung war der Uterus gewöhnlich etwas kleiner und weniger schlaff. Untersuchte man die Patientin längere Zeit, so pflegte sich durch den Reiz der Untersuchung der Uterus zu contrahiren, erschien nach einiger Zeit kleiner und härter. Die Sonde drang meist 9-13 cm tief ein. Die Consistenz des Organs wechselte, wie bereits bemerkt. Wurde kurz vor oder während der Blutung untersucht, so war derselbe immer sehr schlaff. Hatte ich solche Uteri wegen der Blutungen mit starken Chlorzinklösungen etc. geätzt, so war es auffällig, wie schwach die nach der Aetzung aufgetretenen Contractionen waren. Wenn ich solche Uteri excochleirt hatte, so passirte es zuweilen, dass plötzlich starke Blutung auftrat und dass die Curette an verschiedenen Stellen viel tiefer als die Sonde eindrang (bis auf $17 \mathrm{~cm}$ ), was von mir anf die weitere Erschlaffung. des Organs, hervorgerufen durch den Reiz der Curette, bezogen wurde. Die ausgeschabte Schleimhaut schien, makroskopisch betrachtet, einzelne Mal etwas ödematös zu sein, andere Mal erschien sie nahezu normal, Die Secretion aus dem Uterus war bezüglich Menge und Beschaffenheit sehr variabel. Kurz vor und nach den Menses war sie meist rejchlicher als in der intermenstruellen Zeit. Bei der sehr häufig sich findenden chronisehen Metritis der Frauen in den 40 er Jahren war die Secretion in der intermenstruellen Zeit meist gering, bei jüngeren Frauen dagegen war sie häufig beträchtlich. Bei der Totalexstirpation fiel der Umstand zunächst auf, dass die Venen in den Ligamentis latis beträchtlich erweitert waren. Das exstirpirtc Organ war immer vergrössert, zuweilen sehr beträchtlich. So war in Fall 1 der Uterus $14 \mathrm{~cm}$ lang, $5 \mathrm{~cm}$ dick, $11 \mathrm{~cm}$ breit; in Fall 2 (operirt von Klein) war der Uterus kindskopfgross, er wog $250 \mathrm{~g}$; in Fall 4 (Klein) war der Uterus $15 \mathrm{~cm}$ lang; in Fall $611^{1} / 2 \mathrm{~cm}$ lang, $9 \mathrm{~cm}$ breit, $3 \frac{3}{4} \mathrm{~cm}$ dick. In Fall 7 war der Uterus mannsfaustgross ete.

Die Mucosa zeigte makroskopisch keine besondere Abnormität, insbesondere war sie nicht verdickt. Die Messung zeigte, dass sie nicht dicker war als die normale Schleimhaut, mit Ausnahme von 2 Fällen. In einem derselben war jedoch das Organ während der Menses exstirpirt worden. Auf der Schnittfläche fiel stets auf, dass das kranke Organ eine viel weniger rothe Farbe zeigte, als der normale Uterus. Schon mit blossem Auge sah man sehr zahl- 
reiche grane sehnige Fasern, die die Dicke einer Stricknadel und darüber haben, das Organ durchziehen. Auf der Sehnittfläche ragten stets sehr zahlreiche Gefässe hervor, viel zahlreicher als im normalen Uterus.

Mikroskopisch zeigte sich in allen unseren Fällen eine entschiedene Abnahme der Musculatur und Zunahme des Bindegewebes. Das Verhältniss von Bindegewebe zur Muskulatur wechselte am metritisch erkrankten Uterus ebenso wio am normalen an verschiedenen Stellen. Ebenso ist ja auch die Menge des Bindegewebes verschieden, je nach dem Alter der Frau, der Zahl ihrer Geburten u.s.f. (siehe Theilhaber und Meier, dieses Archiv, Bd. 66, H. 1). Aber auch alles dies in Rechnung gezogen, bleibt im metritischen Uterus immer noch eine sehr beträchtliche $\mathrm{Zu}$ nahme des Bindegewebes gegenüber der Reichlichkeit dieses Gewebes im normalen Cterus gleichalteriger Frauen mit annähernd gleicher Anzahl von Geburten.

Die Muskelfelder sind bei metritisch erkranktem Uterus an den meisten Stellen kleiner als beim normalen. Das Bindegewebe, das die Muskelfelder umschliesst, ist dicker; es gelangen dickere und zahlreichere Ausläufer des Bindegewebes in die Muskelfelder hinein, die wieder kleinere, feinere Maschen bilden; an zahlreichen Stellen ist das Muskelgewebe fast vollständig substituirt von Bindegewebe. Daneben findet man zuweilen auch noch spärliche Stellen, in denen Form und Beschaffenheit der Muskelfelder wenig differiren von denen der Muskelfelder des normalen Uterus. - Regelmässig fand sich (in den länger bestehenden Fällen von Metritis) Zunahme der Zahl der Blutgefässe. Dieselbe ist so beträchtlich, dass sie am exstirpirten Uterus leicht schon mit unbewaffnetem Auge constatirt werden kann; unter dem Mikroskop sieht man nicht selten 4-6 Gefässlumina dicht nebeneinander liegen. Die Wandungen der Gefässe waren in den von mir und meinen Assistenten untersuchten Uteris meist dicker als normal. Doch lege ich dieser Anomalie keine Bedeutung bezüglich der Entstehung der Blutungen bei; es handelt sich hier in der Regel nicht um Atheromatose der Gefässe, sondern nach meiner Auffassung um stenosirende Arteriitis, wie sie nach Entbindungen Aborten; und im höheren Alter physiologiseh ist (s. Theilhaber, Die Ursachen der präklimakterischen Blutungen, dieses Archiv, Bd. 62, H. 3).

Fasse ich meine seit Jahren darauthin gerichteten klinischen Beobachtungen und meine hier angedeuteten anatomischen Unter- 
suchungen zusammen, so komme ich zu dem Schlusse, dass es eine ausserordentlich häufig vorkommende Krankheit giebt, bei der der Ausgangspunkt der Affection im Mesometrium zu suchen ist und die anatomisch als bindegewebige Hyperplasie des Uterusparenchyms charakterisirt ist, und die in vielen Punkten dem ähnelt, was Scanzoni und Fritsch als "chronische Metritis" beschrieben haben. Dass diese Krankheit eine primäre Erkrankung des Mesometrium darstellt und nicht durch Erkrankung der Schleimhaut hervorgerufen ist, dafür spricht schon die makroskopische Betrachtung. Mit dem blossen Auge konnte meist gar keine Zunahme der Dicke der Schleimhaut constatirt werden, während andrerseits das Mesometrium hochgradig verändert war; so wog in einem Falle bei nicht verdickter Schleimhaut der Uterus $250 \mathrm{~g}$; er hatte die Grösse eines Kindskopfes. In einem anderen Falle war der Uterus mannsfaustgross bei einer Schleimhaut, deren Dicke nicht die der normalen überstieg. In einem dritten Falle, bei Frau H., war der Uterus $14 \mathrm{~cm}$ lang, $5 \mathrm{~cm}$ dick, $11 \mathrm{~cm}$ breit. Die Schleimhaut war nahezu normal, nur zeigte sich mikroskopisch geringes Oedem derselben. Bei einer anderen Patientin war der Uterus $11 \frac{1}{2} \mathrm{~cm}$ lang, $9 \mathrm{~cm}$ breit, $3 \frac{3}{4} \mathrm{~cm}$ dick, bei einer sehr dünnen Schleimhaut, die ebenfalls in geringem Maasse ödematös war, sonst nichts Abnormes darbot. Variationen in der Dichtigkeit der Drüsen mit Oedem in der Schleimhaut sind doch wohl nicht charakteristisch für eine echte Endometritis, und ich glaube, dass die Zahl der Drüsenlumina in einem Schnitt einer Uterusschleimhaut sehr variabel ist, je nach dem Alter und dem Ernährungszustand bei Patientinnen. Das Oedem in der Schleimhaut andererseits dürfte wohl eher als Zeichen der von mir bereits beschriebenen venösen Stauung anzusehen sein. Keinesfalls kann ich annehmen, dass diese Veränderungen primär sind, und selbst wenn sie es wären, wäre es doch kaum glaublich, dass solch geringgradige Veränderungen der Mucosa zu so hchgradigen Anschwellungen und Degenerationen des Mesometriums führen können. Letztere müssen ganz entschieden als unabhängig von der sogen. Endometritis angesehen werden.

Die chronische Metritis ist also charakterisirt durch Abnahme der Muskulatur und Zunahme des Bindegewebes im Mesometrium bei gleichzeitiger Vergrösserung des Organs, Erweiterung seiner Höhle und gleichzeitiger starker Blutfüllung des Uterus. Die Zunabme des Bindegewebes im Uterus, "bindegewebige Hyperplasie" 
dieses Organs, ist zwar auch ein physiologischer Process (in den präklimakterischen Jahren und nach dem Klimakterium z. B.). Allein in dieser Zeit pflegt das Organ in normalen Fällen nicht grösser zu werden, sondern im Gegentheil zu atrophiren, der Uterus ist in dieser Zeit nicht hyperämisch, sondern im Gegentheil anämisch. Sobald es jedoch (auch in der präklimakterischen Zeit) bei der Atrophie der Musculatur und bei der Zunahme des Bindegewebes zu starker Hyperämie im Uterus kommt, wird sich venöse Stauung einstellen, da eben in Folge der Abnahme der Muskulatur der Uterus "insufficient" ist, d. h. da seine Contractionen zu schwach sind, um dazu beizutragen, das Venenblut zum Herzen zu treiben. Die venöse Stauung führt dann allmälig im Laufe von Monaten oder Jahren zur Vergrösserung des Organs.

Würde man gleich im Beginn der Erkrankung einen solchen Lterus exstirpiren und anatomisch untersuchen, so würde man wohl sehr häufig am herausgeschnittenen Organ Zeichen der Hyperämie vermissen, da ja die Hyperämie zunächst meist durch vasomotorischen Einfluss hervorgerufen ist, also durch die Contractionen der Gefässe am herausgeschnittenen Organ sich der Beobachtung entzieht. Nach langem Bestehen der Krankheit hat jedoch die Hyperämie häufig zu einer dauernden Dilatation der Gefässe geführt. Deshalb wird man dann, wenn man den Uterus exstirpirt, meist reichliche Bindegewebsentwicklung in demselben finden und in der That vermissten wir niemals eine starke Entwicklung der Blutgefässe an den von Klein und mir exstirpirten. Uteris.

Was die Ursachen der ehronisehen Metritis betrifft, so findet man namentlich bei älteren Autoren die Angabe, dass sie sich ziemlich oft aus der acuten Metritis herausbilde. Nach meinen Beobachtungen glaube ich, dass dies recht selten der Fall ist. $A b$ und $z u$ kommt es zu einer mässigen Anschwellung des Uterusparenchyms bei langdauernder gonorrhoischer Erkrankung des Uterus, hie und da auch einmal giebt die puerperale Metritis Veranlassung zu einer dauernden Bindegewebshyperplasie des Organs; doch sind dies nur bei einem recht kleinen Procentsatz der zur Beobachtung kommenden Fälle von Metritis die Ursachen.

Dass Flexionen und Versionen des Uterus ehronische Metritis verursachen, glaube ich nicht. Meines Erachtens handelt es sich hier nur um zufällige Complicationen oder es hat die Perimetritis, die die Dislocation bez. Fixirung des Uterus herbeigeführt hat, gleichzeitig auch die Sehwollung des Uterusparenchyms nach 
sich gezogen (s. Theilhaber, die Retroflexio uteri, Monatssehr. f. Geb. Bd. 2 u. 3 u. Verhandlung des 7. Congresses d. deutschen Ges. f. Gyn.)

Ebenso halte ich es für unwahrscheinlich, dass der Prolaps eine echte Metritis des Uteruskörpers hervorruft. Es scheint sich hier nur um Elongation der Cervix durch den Zug zu handeln.

Vielfach wurde auch behauptet, dass Geschwülste (Myome, Carcinome u. s. f.) Metritis, d. h. bindegewebige Hyperplasie des Uterus machen. Ich habe durch meine Assistenten Ant. Meier, Hollinger und von Lorentz eine grössere Anzahl myomatöser und carcinomatöser Uteri nach dieser Richtung hin untersuchen lassen. Das Ergebniss dieser Untersuchungen wird demnächst publicirt.

Wir können uns nicht der Ansicht anschliessen, dass diese Tumoren wirklich Metritis verursachen.

Eine der häufigsten Ursachen der chronischen Metritis ist die beginnende Klimax. Untersucht man häufig Frauen in der präklimakterischen Zeit nach dieser Richtung hin, so ist man erstaunt, wie ausserordentlich häufig man einen wesentlich vergrösserten, dicken, meist schlaffen, manchmal aber auch harten Uterus findet mit weiter Uterushöble. Reizung der Uterusmucosa. ruft keine oder nur sehr geringe Reaction hervor. Die Curette führt oft zu hochgradiger Erschlaffung dieses Organs. Der Uterus ist gerade in diesen Jahren häufig so gross, dass man im Zweifel ist, ob man nicht einen Uterus myomatosus vor sich hat. Der Grund der Entstehung der chronischen Metritis in diesen Jahren liegt meines Erachtens in einem Schwund der Musculatur, der eine regelmässige Begleiterscheinung der herannahenden Senescenz. der Genitalien ist. Physiologischer Weise geht parallel mit diesem Schwund der Musculatur eine Stenosirung der Gefässe, eine Verringerung der Blutmasse, die zum Uterus strömt. Bleibt diese Verengerung der Gefässe aus irgend welchen Gründen aus (Excesse in venere, üppige Lebensweise u. s. f.), so kommt es in Folge der Muskelinsufficienz zu venöser Stauung; die Folge ist die Hyperplasie des Organs, "chronische Metritis", in einzelnen Fällen vielleicht begleitet von secundärer Schwellung der Schleimhaut, hervorgerufen durch venöse Hyperämie und Oedem, "fungöse Endometritis". Diese Form "Metritis praeclimacterica" ist nach meinen Beobachtungen die häufigste Ursache der chronischen Metritis; ihr gewöhnliches Symptom sind die präklimakterischen Blutungen. Durch zuneh- 
mende Stenosirung der Gefässe (in den nächsten Jahren) können die Blutungen spontan sistiren, man findet dann oft noch den grossen Uterus, ohne dass mehr Menorrhagien vorhanden sind. Beobachtet man solche Patientinnen jahrelang, so sieht man wie nach Jahren nach dem Verschwinden der Menorrhagien (in der postklimakterischen Zeit) der Uterus dann allmählich kleiner und atrophisch wird, meist später in Retroflexion fällt; dies wäre also dann das zweite Stadium der chronischen Metritis nach Scanzoni, das allerdings dann meist keine Symptome mehr macht. Während aiso vermehrte Blutungen bei der präklimakterischen Metritis sehr gewöhnlich sind, ist vermehrter Ausfluss bei dieser Form nicht sehr häufig, vermuthlich weil oft die Zahl oder die Functionen der Drüsen bereits durch die herannahende Senescenz vermindert sind.

Es darf jedoch nicht übersehen werden, dass im ersten Stadium, d. h. nach kurzem Bestande der venösen Stauung bei den präclimacterischen Blutungen der Uterus nicht selten auch klein, seblaff, atrophisch ist, dass es also auch präklimakterische Blutungen bei kleinem Organ und bei geradezu atrophischer Schleimhaut giebt. -

Die chronische Metritis findet sich auch nach längerem Bestande von Adnexerkrankungen, namentlich nach lang dauernder subacuter und ebronischer Perimetritis, wie sie vor Allem bei Salpingitiden und ibren Folgeerscheinungen nicht selten sich einstellen. Auch für diese Art der chronischen Metritis gelten in Wirklichkeit die beiden Formen, die Scanzoni s. Zt. für die chronische Metritis im Allgemeinen geschildert hat: Auch hier fand jeh bei Laparotomien im ersten Stadium, d. h. nach kürzerem Bestande der Erkrankung, das Organ gross, weich, dick, hyperämisch schlaff. Nach jahrelanger Dauer der Erkrankung konmt es zur Abnahme des Blutgehaltes, zur Verkleinerung und Verbärtung des Uterus. Die Metrorrhagien pflegen hier im ersten Stadium vorhanden zu sein, später wenn die Perimetritis nachlässt und der Uterus seine Schlaffheit verliert, wenn er hart und derb wird, pflegen die Menses sich zu reguliren.

Häufige Wochenbetten disponiren ebenfalls zur chronischen Metritis durch Atonie der Uterusmusculatur; denn der multipare Uterus ist stets reicher an Bindegewebe als der nullipare. Dementsprechend finden wir auch bei Frauen, die viele, namentlich schnell aufeinanderfolgende Wochenbetten gehabt haben, nicht selten Vergrösserung des Uterus, Menorrhagien, Fluor. Man kann 
im Allgemeinen sagen, dass die Anzahl der Geburten in gewissem Verhältniss steht zur Grösse des Uterus. Es ist möglich, dass durch die Entbindungen selbst, es ist möglich auch, dass durch Störungen im Wochenbett Musculatur verloren gegangen ist; überall da, wo specifische Gewebselemente durch Entzündung zu Grunde gehen, bildet sich zum Ersatz Bindegewebe. Ausserdem dürfte auch ein Teil des Bindegewebes aus den zahlreichen Gefässen des hochschwangeren Uterus stammen, welche im Wochenbett z. Th. in solide Stränge umgewandelt werden. Diese Verminderung der Vlusculatur und Vermehrung des Bindegewebes nach häufigen Entbindungen erklärt uns nicht bloss, wie bereits angeführt, den Umstand, dass solche Frauen sehr häufig an Vergrösserung des Uterus, Menorrhagien und Fluor leiden; sie erklärt auch die Thatsache, dass Wehenschwäche bei Frauen, die häufig entbunden haben, so oft beobachtet wird. Freilich macht sich diese bei der Ausstossung des Kindes zuweilen nicht bemerkbar, denn da andererseits der Widerstand der Weichtheile im Becken um so kleiner wird, je zahlreicher die Entbindungen varen, so geht die Ausstossung des Kindes trotz der Verminderung der Muskelmasse des Uterus häufig noch leicht von Statten. Dagegen kommt die Vorringerung der Muskelmasse in der Nachgeburtsperiode und nach Ausstossung der Nachgeburt zur Geltung. Es ist allgemein bekannt, dass atonisehe Zustände in dieser Zeit mit Retention der Placenta oder auch mit Blutungen nach Ausstossung derselben sich recht häufig ereignen, wenn die Frau oft entbunden hatte. Man findet in solchen Fällen einen auffallend schlaffwandigen Uterus, der auch nicht allzu selten zu partiellen oder totalen Inversionen neigt. -

Auch Ueberanstrengung des Uterus bei engem Becken scheint eine Prädisposition zu dieser Muskelatrophie und Bindegewebshyperplasie zu geben; denn in solchen Fällen sind die geschilderten atonischen Zustände des Uterus während und nach der Entbindung ganz besonders häufig.

Myodegeneratio ateri in Folge von Chlorosis habe ich ziemlich hänfig beobachtet. Zuweilen entstehen durch dieselbe schon während des Bestandes der Bleichsucht die Menorrhagien. In der Mehrzahl der Fälle jedoch kamen die Blutungen erst nach Ablauf der Bleichsucht zum Ausbruch. Es ist eben häufig die Congestion zum Uterus in Folge der Verringerung der Blutmenge während der Bleichsucht vermindert. Doch kann eine Hyperämie 
in den Genitalien auch während dieser Erkrankung vorhanden sein. Dann ist die schwache Uterusmuskulatur nicht im Stande den Blutlauf in der Gebärmutter genügend zu reguliren, es kommt dann zu Blutung oder zu Ausfluss. Nanchmal jedoch stellt sich diese Hyperämie erst ein nach Ablauf der Chlorose. Wenn dann die Muskulatur noch nicht geniigend regenerirt ist, findet man oinen grossen dicken Uterus bei oft sonst virginalen Verhältnissen.

Aehnliche Verhältnisse wie bei Chlorose findet man auch bei Infiltration der Lungen und bei anderen sehweren Krankheiten wie Typhus, Gelenkrheumatismus oder in der Reconvalescenz von solchen Krankheiten (s. Theilhaber und Meier l. c.). Auch bei sehr jungen $\mathrm{Mädchen} \mathrm{finden} \mathrm{sich} \mathrm{zuweilen} \mathrm{solche} \mathrm{metritische}$ Anschwellungen. Es sind danu regelmässig starke Blutungen vorausgegangen, die dann offenbar zunächst durch eine ungenügende Entwicklung der Muskulatur des Uterus bedingt waren, letztere hatte zu venöser Stauung und zu späterer Anschwellung des Uterus geführt.

Ebenso wie die Perimetritis kann auch langdauernde Parametritis zu collateraler Hyperämie mit Stauung im Uterus führen, dadurch kann es denn auch zu Hyperplasie des Organs kommen, zur chronischen Metritis. In diesem Falle tritt meist Spontanheilung an, wenn die Parametritis abgelaufen ist. Die abnormen Blutungen schwinden, der Uterus wird wieder kleiner; hat jedoch der Process sebr lange bestanden, so kann auch eine mässige Anschwellung des Organs noch viele Jahre bestehen bleiben, die dann meist keine Symptome macht.

Das Woehenbett als solehes kann ebenfalls eine Ursache der chronischen Metritis (bei schlechter Entwicklung der Muskulatur) sein. Nach meinen Beobachtungen ist der Entwicklungsgang in der Regel folgender: Die in manchen Fällen relativ zu schwache Uterusmuskulatur ist nicht im Stande das noch reichlich im Uterus circulirende Blut genügend zum Herzen zurückzutreiben, es entsteht dadurch venöse Stauung, Ueberernährung des Organs, Bindegewebshyperplasie, Stauungen in der Schleimhaut und manchmal Oedem derselben. Es sind dies wohl dieselben Fälle, die von den Autoren Einn, Klebs u. s. w. untersucht worden waren, und die bei ihnen die Meinung hervorgerufen hatten, es kämen bei der chronischen Metritis auch Wucherungen der Muskulatur vor, denn in der That muss man $\mathrm{ja}$ in solchen Fällen reichliches Vuskelgewebe finden, wenn man nicht zn lange nach der Entbindung zu untersuchen 
Gelegenheit hat. Es ist jedoch hier die Reichlichkeit der Muskulatur nicht eine Folge von Neubildung derselbon, sondern die Reichlichkeit der Muskulatur ist nur noch eine Folge des noch nicht vollständig abgelaufenen Puerperiums.

Dass allgemeine Circulationsstörungen (in Folge von Herzleiden etc.) eine Ursache der chronischen Metritis seien, ist eine Angabe, die man ebenfalls in vielen älteren Lehrbüchern findet. In Wirklichkeit kommt dies klinisch recht selten zur Beobachtung, vielleicht deshalb, weil die Compensationsstörungen des Herzens oft doch erst zu einer Zeit sich einstellen, in der die Frauen schon die Klimax hinter sich haben. Dagegen ist es für mich ausser Zweifel, dass Excesse in Venere, ebenso wie Onanie und allgemeine Plethora Hyperämien im Uterus herbeiführen und dadurch mit Veranlassung zur Entstehung der Metritis chronica geben können.

Die Symptome der chronischen Metritis sind vor allem Blutungen und Ausfluss. Die starke Blutungen sind die Folge der venösen Stauung und des Umstandes, dass die atrophische Uterusmusculatur sich während der Menses ungenügend contrahirt, in Folge davon werden die sehr zahlreichen zerrissenen Blutgefässe nicht genügend comprimirt. Das Auftreten der Blutungen ist ein sehr verschiedenes: Die stärksten und längstdauernden Blutungen beobachtete ich bei der präklimakterischen Metritis. Hier halten die Blutungen nicht allzuselten eine Reihe von Wochen, ja sogar von mehreren Monaten ununterbrochen an. Ich sah Metrorrhagien, die beim Eintritt in meine Behandlung bereits $4 \mathrm{ja}$ 5 Monate fortgedauert hatten. Charakteristisch für die präklimakterische Metritis ist es, dann dass zuweilen wieder eine mehrmonatliche Amenorrhoe sich einstellt. In der Zeit der Amenorrhoe ist (zum Unterschied von Corpuscarcinom) häufig die Frau vollständig frei von jeglichem Ausfluss.

Auch bei der juvenilen Form der Metritis sieht man zuweilen Blutungen, die eine ganze Reihe von Wochen anhalten. - Blutungen von mehrwöchentlicher Dauer sah ich auch nicht selten bei der Metritis, wie sie sich bei Adnexerkrankungen einstellt, also bei der Perimetritis in Folge von Salpingitis und bei Tubenmolen, beim incompleten Tubarabort etc.

Der Ausfluss ist im Allgemeinen spärlich bei älteren Frauen; bei der präklimakterischen Metritis ist er meist gering oder fehlt ganz, dagegen ist er häufig bei jüngeren Frauen in der inter- 
menstruellen Zeit recht reichlich. Wenn beim "Adnexuterus" reichlicher Ausfluss vorhanden ist, so ist er natürich nicht immer auf die Metritis allein zurückzufübren, sondern häufig ist er wohl mit verursacht durch eine Endometritis gonorrhoica.

Schmerzen macht nach meinen Beobachtungen die chronische Metritis nicht. Wo solche vorhanden waren, konnte ich immer eine complicirende Perimetritis nachweisen, oder die Schmerzen waren durch irgend eine andere Complication (Hysterie, Darmerkrankung u. s. f.) verursacht. Auch besondere Empfindlichkeit bei Druck vermisste ich stets bei der uncomplicirten Metritis. Auch die sogenanten reflectorischen Symptome, Kopfschmerz, Schwindel, Flimmern vor den Augen, Neuralgien im Gesicht, auf der Brust u. s. f. konnte ich niemals auf die chronische Metritis als solche beziehen. Ebenso wenig glaube ich, dass die Dysmenorrhoe oder die Sterilität mit der chronischen Metritis etwas zu thun haben. Conception ist bei dieser Erkrankung sicherlich nicht etwas seltenes, vorausgesetzt, dass die Tuben und Ovarien nicht anderweitig erkrankt sind.

Die Grösse des Uterus ist, wie bereits angeführt, variabel je nach der Dauer der Erkrankung, je nach den veranlassenden Ursachen etc. In der Mehrzahl der Fälle findet sich eine nicht unbeträchtliche Vergrösserung des Uterus.

Regelmässig finde ich bei der chronischen Metritis eine Erweiterung der Uterushöhle.

Was die Veränderungen der Vaginalportion betrifft, so habe ich sie in dieser Abhandlung niemals erwähnt. Meine Untersuchungen erstreckten sich bis jetzt nur auf die Veränderungen des Corpus uteri. Von den Erkrankungen der Vaginalpartion, Erosion, Eetropium, Verdickungen derselben u. s. w. glaube ich, dass sie mit der eigentlichen "Metritis chronica" in der Regel nicht im Zusammenhang stehen. Die Vaginalportion ist physiologisch und histologisch eigentlich mehr als ein Abschnitt der Scheide, denn als ein solcher des Uterus zu betrachten und ihre Erkrankungen sind häufig unabhängig von den Erkrankungen des Uteruskörpers.

Was die Behandlung der chronischen Metritis betrifft, so ist sie fast in allen Punkten identisch mit der Behandlung ihres Hauptsymptoms, der Insufficienz. Die zahlreichen, uns gegen letztere zur Verfügung stehenden Mittel habe ich in der Münch. med. Wochenschr. 1902. No. 42 registrirt.

kis erübrigt mir noch, auf Grund unserer neuesten Unter- 
suchungen einige Worte bezüglich der Bedeutung der Excochleation zu sagen. Wenn auch auf Grund unserer letzten Forschungen die Bedeutung des Endometriums für die Entstehung der Blutungen noch weiter reducirt wird, so folgt daraus noch nicht, dass dic Ausschabung aus der Therapie der chronischen Metritis vollständig zu streichen ist. Empirisch lässt sich doch feststellen, dass neben vielen Misserfolgen auch zuweilen Besserungen durch die Excochleation erzielt werden. Freilich ist es schon lange bekannt, dass zur Erzielung von Heilungen meist noch eine Reihe von Aetzungen der Uterusschleimbaut nach der Ausschabung nothwendig sind. Ein grosser Theil der erreichten Erfolge ist also vielleicht häufig den durch die Aetzung verursachten Uteruscontractionen zuzuschreiben. Andererseits sehen wir häufig, dass die erste Menstruation nach der Ausschabung mit sehr starker Blutung einhergeht. Letzteres harmonirt nicht recht mit der Idee, dass durch die Ausschabung die Krankheitsursache beseitigt sei.

Es ist ja möglich, dass die Ausschabung auf andere Weise bei abnormen Uterusblutungen nützt, etwa durch die mit derselben verbundene Blutentziehung, die ja erfahrungsgemäss ein gutes Mittel gegen venöse Hyperämien ist.

Früher wurden ₹on den Gynäkologen sehr häufig und mit sehr guten Erfolgen Blutentziehungen an der Portio als Mittel gegen "die Blutungen bei chronischer Metritis" angewandt (s. die älteren Auflagen von Schroeder's Gynäkologie, das Lehrbuch von Scanzoni ete.).

Vielleicht wirkt auch die Ausschabung manchmal durch Eröffnung einer grösseren Anzahl von Blutgefässen in der Mucosa, Veranlassung von Thrombenbildung in derselben, wodurch die Circulation im ganzen Uterus wieder beeinflusst wird.

Auch die mit der Ausschabung verbundene Uterusausspülung kann zuweilen von Nutzen sein,, da der durch dio Ausspülung ausgeübte Reiz auf der nackten Musculatur besonders energisch zu Contractionen Veranlassung giebs. 